\title{
REAL TIME RUT PROFILE MEASUREMENT IN VARYING TERRAIN TYPES USING DIGITAL IMAGE CORRELATION
}

\author{
Theunis Botha ${ }^{\mathrm{a}, *}$, Devin Johnson ${ }^{\mathrm{a}}$, Schalk Els ${ }^{\mathrm{a}}$, Sally Shoop ${ }^{\mathrm{b}}$
}

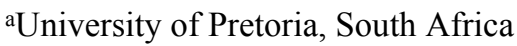

${ }^{\mathrm{b} C}$ Cold Regions Research Laboratory, USA

*Corresponding author. E-mail address: theunis.botha@up.ac.za (T. Botha).

\begin{abstract}
An important parameter in terramechanics is the rut depth produced when a vehicle traverses deformable soil. The rut depth provides a measure of vehicle tractability as well as the impact on the environment. Rut depth is not uniform on natural terrain and typically only a few manual points are measured. Synchronizing rut depth with other measurements is also problematic.

This paper investigates the feasibility of using cameras to measure 3D terrain profiles from which a single rut depth measurement is obtained. Tests were performed on different vehicles, for various dynamic vehicle manoeuvres, over varying terrains including sand, mud, grassland, snow and ice. Results were validated using the traditional stick ruler method.

Measurement frequencies of $58 \mathrm{~Hz}$ were obtained using affordable commercially of the shelf computational hardware and dedicated software. Determining whether a vehicle can traverse a terrain can significantly improve the vehicle mobility. Therefore, real time measurements of rut depth can be used to determine the mobility of vehicles in off-road conditions that can change rapidly due to environmental conditions e.g. rain or snow. The techniques described can assist in gathering terrain and vehicle mobility data that can be used directly to assist the driver in making safety related decisions.
\end{abstract}

Keywords: Real time; Rut depth; Stereo; Terrain measurement

\section{Introduction}

The traversing of vehicles over deformable soils results in the compaction of the soil and the formation of ruts. The soil compaction and rut formations have a significant effect on the ability of vehicles to traverse the terrain and the impact of the vehicle on the terrain. The soil strength and rut depth can be used to determine whether a vehicle can safely traverse the terrain without the vehicle becoming immobilized. The formation of ruts also causes water to pool in these areas which in cold conditions results in the formation of ice on the surface which effects vehicle traction. Therefore, the rut profile is an important parameter which could be beneficial to 
vehicle mobility if it can be measured in real time with ease and accuracy. Rut measurements of multiple passes over the same terrain can assist in determining eventual sinkage levels where multiple vehicles pass through the same ruts.

Soil strength is often measured using a cone penetrometer or Bevameter, however the tests are conducted statically and cannot be used to measure the soil strength in real time. Herrick and Jones (2002) however conducted tests on clay loam soil and found a relationship between the soil sinkage and the penetration resistance, dynamic drop-cone resistance and pre-compaction stress. While the relationship between soil sinkage and soil strength are vehicle dependent, a relationship for a specific vehicle can be obtained. This allows soil strength to be measured based purely on soil sinkage which is easier to measure in realt-time on a vehicle. Hemmat et al (2014) also showed that a relationship between soil mechanical properties and rut depth can be determined for certain soil types.

In rural conditions, where there are no permanent roads, any passage by vehicles could affect future trafficability. Constant assessment of the road is necessary especially if the road has a deformable surface such as snow, mud, or un-compacted sand. The effect that heavy vehicles have on these deformable terrains is so substantial that several studies have been conducted to reduce the effect of heavy vehicles trafficking over deformable terrains (Shoop et al., 2013), (Halvorson et al., 2001). Soil rut depth is also a useful measure, due to its simplicity, of the amount of soil compaction (Soane et al., 1980). Soil compaction can lead to soil degradation by enhancing harmful physical, chemical and biological processes, which may affect the energy needed for crop production (Soane and van Owerkerk, 1995). Anderson et al. (2005) also uses the rutting of terrains to assess the level of vehicle impact on the environment. Therefore, the development of a sensor to automatically and accurately measure soil deformation would lead to better energy efficiency not only in the vehicles themselves but also the in the production of crops.

In terramechanics soil deformation is not only used to evaluate the performance of wheels in deformable terrain (Shibly et al., 2005) but also used to improve soil models for simulation and prediction (Iagnemma et al., 2003). Accurate measurement of the deformed soil can improve the development of off-road terrain wheels, which would lead not only to improved tractive performance, but would also mitigate the rutting of soils. The rutting of soils also increases the rolling resistance of tyres, which is known as motion resistance (Kurjenluoma et al., 2009), thus decreasing vehicle efficiency.

Therefore, the real time measurement of the rut profile can be used to aid in vehicle performance. Vehicle performance can be increased by first assessing the soil strength and determining whether the vehicle can negotiate 
the terrain, thus reducing the chance of immobilization. Road conditions can also be assessed by vehicles in a platoon. When the road conditions have deteriorated to such an extent than an alternative road should be used, information can be relayed from vehicle to vehicle. The effect of vehicle control systems such as tyre pressure inflation control or traction control can be assessed in real time to improve vehicle performance. Continual assessment of the road conditions and the impact of a vehicle on the terrain can also be used to evaluate the need of road maintenance.

Rut profile measurements are typically conducted using a ruler. In Shoop et al. (2013) a single point at the maximum rut depth is taken as a measurement. The rut profile is often measured using multiple rulers and takes an image of the result for post processing (Halvorson et al., 2001), (Shoop et al., 2008). These techniques are used due to their low cost. A LiDAR based rut measurement system by Salmivaara et al (2018) showed that the technique can be used to determine rut depth and found a RMSE accuracy of less than 35mm. Photogrammetry is another photographic method whereby multiple overlapping images are used to determine depth (Pierzchała et al. 2014, 2016 . In particular Kenarsari et al (2017) used a system where three cameras were placed after a tractor tyre to determine the rut created by a tyre. The system can also be placed on an Unmanned Ariel Vehicle (UAV) to record larger areas (Siebert and Teizer, 2014). Photogrammetry can provide very accurate results but often require photos from multiple angles to obtain good results which adds complexity. The major drawback however is that the method is rather computationally expensive and has a scale ambiguity therefore the method can't be used in real time.

The major problem with most current techniques are that they are either very expensive to implement, using LiDAR, or the method is too slow to be used for real time measurements such as photogrammetry. The need for accurate real time results which can possibly used to determine the mobility of a vehicle of deformable soil required.

In this study, rut profile measurements were conducted using inexpensive off the shelf cameras to create a calibrated stereo measurement system which has no scale ambiguity. As the need for the system is to accurately measure the rut in real time, the focus of the study is on stereovision based algorithms which can offer real-time results. The computational speed and accuracy of each algorithm is determined as there is generally a trade-off between computational speed and accuracy.The system is evaluated on unprepared, undisturbed as well as trafficked snow, ice, mud, sand and grass terrains. The stereo based system has been used in previous studies to successfully conduct vehicle measurements such as slip-angle (Botha and Els, 2015a), longitudinal slip (Botha and Els, 2015b) and road profiling (Botha and Els, 2015c) in post processing. 


\section{Determining Rut Profile Through Measuring Road Texture}

The method used to obtain depth from multiple cameras is very similar to one of the ways a human captures depth through the use of two eyes. Stereovison works by determining the coordinate difference $\left(x_{l}-x_{r}\right)$ of an object or region in two images (see Figure 1). This measurement is commonly referred to as disparity. Thus, the main problem to solve in order to obtain 3D coordinates from 2 cameras is the problem of matching regions (disparity mapping) between two images. The disparity map between two images from a calibrated rig can then be used to determine 3D location of regions in the image relative to the cameras. The cameras are calibrated to remove lens distortion and to obtain the relative translation and rotation between the cameras. The calibration process also removes any scale ambiguity and therefore unlike photogrammetry this process does not require scaling. The disparity mapping is usually achieved by using a correlation metric and performing a searching across the two images. Performing an exhaustive global searching can be very computationally expensive. Therefore, epipolar geometry, which maps a feature projected onto one image as a line on the other image, is used to reduce the global search to line searches thereby vastly improving computational efficiency. The epipolar geometry is obtained from the calibration process and the result of a rectified image pair is shown in Figure 1 . The figure shows that a feature on the left image lies on the same horizontal line in the right image.

Various disparity mapping algorithms exist, however, in the aim for real time measurements only computationally efficient algorithms are investigated. Two algorithms of note are the Block Matching (BM) (Konolige, 1997) and Semi Global Block Matching Algorithm (SGBM) (Hirschmuller, 2005). The BM algorithm is one of the most simplistic algorithms and uses a simple search window and correlation metric to find the corresponding pixel in the right camera for each pixel in the left camera. Each pixel search is treated independently of one another, which often results in more discontinuous surface profiles. SGBM is an efficient strategy for minimizing a global energy that comprises of a small window matching cost and pair-wise smoothness terms. It forces a similarity between neighbouring searches enforcing a semi-global search strategy. The algorithm also uses a small matching window to calculate correspondence between the same region occurring in different images. The correspondence match is used in an optimization algorithm that minimizes the energy of a smoothness function. The solution is therefore a point which correlates well between the two images based on the pixel illumination but also reduces the smoothness of the disparity in the region. The smoothness is ideally optimized over the 2D problem, however, to reduce computational complexity the 2D smoothness function is estimated using the aggregate of multiple $1 \mathrm{D}$ smoothness functions. 
For the correspondence matching to work requires that the surface captured using the cameras have visual surface texture which shows up as illumination variation on the images. It is reasonable to assume that a tarred or gravel surface will have texture, however this study will evaluate surfaces that do not show obvious surface texture such as snow, ice and mud.

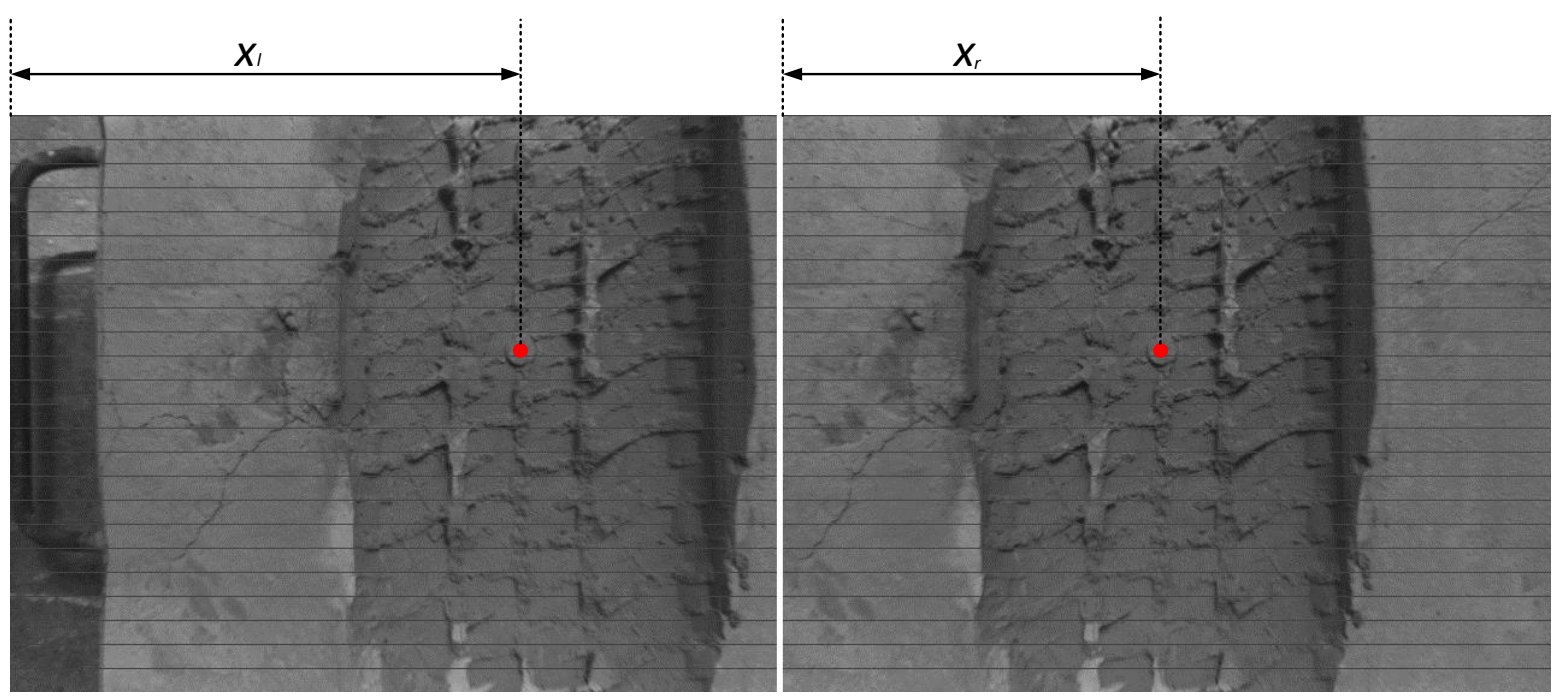

Fig. 1: Rectification of left and right images of rut showing feature in both images

\section{Algorithm Comparison}

The accuracy and computational speed of several implementations of SGBM and BM algorithms are compared to determine the suitability for real time measurements. The following algorithms are compared.

- $\quad$ BM CPU: algorithm from the open source OpenCV library is used. This algorithm provides subpixel matching accuracy up to $1 / 16^{\text {th }}$ of a pixel

BM GPU: algorithm from the open source OpenCV library is used. This algorithm provides subpixel matching accuracy up to $1 / 16^{\text {th }}$ of a pixel

- $\quad$ SGBM CPU: algorithm from open source OpenCV library is used. This algorithm provides subpixel matching accuracy up to $1 / 16^{\text {th }}$ of a pixel

- $\quad$ SGBM GPU CUDA: GPU implementation of the SGBM algorithm from (Hernandez-Juarez et al., 2016) boast high computationally efficiency but does not provide sub-pixel accuracy. The number 
of optimization directions can also be changed between 2, 4 and 8 directions which affect the accuracy and computational efficiency. The algorithm is developed on graphics card developers, NVidia, own parallel computing interface (CUDA) which enables easy use of Nvidia graphics card for general computing.

\subsection{Computational Speed}

The algorithms were implemented on a mid-range i5-570 (2.7GHz) CPU or an entry level GTX840M GPU. The frequency of computation was determined performing the depth computation of images of size $640 x 480$ pixels. The computational efficiency results are shown in Table 1. The results show that the SGBM CUDA 2 pass directions is by far the most computationally efficient algorithm and as the number of optimization directions increase the computational frequency decreases. While the BM GPU algorithm and the SGBM CUDA 8 pass algorithm provided similar results the SGBM CUDA algorithm was found to scale much better with increase in GPU performance. The SGBM CUDA algorithm has been shown that on high end GPU (NVidia Titan X) to achieve speeds of $886 \mathrm{~Hz}, 475 \mathrm{~Hz}$ and $237 \mathrm{~Hz}$ for the 2, 4 and 8 pass directions respectively (Hernandez-Juarez et al., 2016), while the BM GPU does not scale as well with increase in GPU performance.

All of these options are feasible for real time rut depth measurement. Even the slowest $(4 \mathrm{~Hz})$ is expected to provide very useful data for all intended applications

Table 1: Computational frequency of algorithms

\begin{tabular}{|l|l|l|l|}
\hline Algorithm & $\begin{array}{l}\text { CPU mid Range (i5- } \\
\mathbf{5 7 0}\end{array}$ & $\begin{array}{l}\text { GPU entry level (Nvidia } \\
\text { GTX840M) }\end{array}$ & $\begin{array}{l}\text { GPU High End } \\
\text { (NVidia Titan X) }\end{array}$ \\
\hline BM & $8 \mathrm{~Hz}$ & $15 \mathrm{~Hz}$ & Not tested \\
\hline SGBM & $4 \mathrm{~Hz}$ & Not tested & Not tested \\
\hline SGBM CUDA & Not tested & $58 \mathrm{~Hz}$ & $886 \mathrm{~Hz}$ \\
\hline SGBM CUDA & Not tested & & $475 \mathrm{~Hz}$ \\
\hline 4pass & & $30 \mathrm{~Hz}$ & \\
\hline
\end{tabular}




\begin{tabular}{|l|l|l|l|}
\hline SGBM CUDA & Not tested & $16 \mathrm{~Hz}$ & $237 \mathrm{~Hz}$ \\
8pass & & & \\
\hline
\end{tabular}

\subsection{Relative Accuracy of Matching algorithms}

The accuracy of the measurement algorithms is determined by using a reference rut, from a SUV tyre, cast in concrete. A point cloud of the concrete rut was obtained by measuring the surface in a Computer Numerical Control (CNC) lathe using a road profiling laser (Acuity AR700-8) with an accuracy of 50 $\mu$ m as specified by the manufacturer. The X-Y resolution of the CNC was set at $0.1 \mathrm{~mm}$, with the height measured using the road profiling laser. The rut shown in Figure 2 has a rut depth of $40 \mathrm{~mm}$ and the size of the point cloud is $280 \mathrm{x} 450 \mathrm{~mm}$. A point cloud is also generated from photos taken by a calibrated stereo rig comprising of 2 PointGrey Flea3 cameras which have a maximum resolution of 1240x1024 pixels. The PointGrey Flea3 cameras are small (30x30mm) machine vision cameras with a USB3 interface. The cameras are mounted on an aluminum plate 80mm apart and $300 \mathrm{~mm}$ from the surface. The error of the obtained camera surface to the laser measured reference surface is determined by initially aligning the point clouds manually and then using a fine registration technique of Iterative Closest Point (ICP) (Besl and McKay, 1992). The error between the laser based reference and camera measured surface can be obtained as the distance between closest points of the two point clouds. However, since the reference surface has a horizontal accuracy of $0.1 \mathrm{~mm}$ this could negatively affect the measurement. Therefore, a mesh of the reference surface is created. The mesh creates a surface which can effectively be used for interpolation and provide a better estimate of the actual error. The distance between a point on the measured point cloud and the reference mesh is computed. This distance between a measured point and the reference mesh is the error as a point on the mesh will have a distance of zero. The whole process is performed in CloudCompare. A histogram of the error of all the points is created for each measurement algorithm and is shown in Figure 3. A normal distribution is applied to each error histogram and the mean and standard deviation of the error is shown in Table 2.

For the BM and SGBM algorithms the process is conducted on the full pixel resolution of 1MP, as well as, 640x480 pixels (0.3MP). Figure 3shows that the BM and SGBM algorithms provide similar results at both resolutions and, as expected, the accuracy reduces when the resolution is decreased. At 640x480 pixels, the block matching algorithm provides the best accuracy which is slightly less than SGBM. The fast SGBM CUDA 
algorithm shows similar mean to the BM algorithm however the standard deviation is significantly larger and increases as fewer optimization directions are used. The reason for the SGBM CUDA algorithms having larger standard deviation is due to the lack of sub-pixel accuracy. This results in the resolution of the algorithm being limited which increases the average error.

Thus, the rut profile can be measured with most points within a $1.5 \mathrm{~mm}$ of the reference at $15 \mathrm{~Hz}$ using the GPU BM algorithm at a resolution of 640x480 pixels. The speed would be increased by using more powerful GPU. If the accuracy is to be determined within $5 \mathrm{~mm}$ then the CUDA SGBM algorithm can be used at $60 \mathrm{~Hz}$ on a low cost GPU and scales very well as the GPU performance is increased. Added speed can be obtained by performing the depth estimation on a smaller longitudinal section i.e. 20x480 pixels. The smaller images would still capture the whole rut laterally but not fully in the longitudinal direction. In order to determine the depth the complete rut in the longitudinal direction need not be determined as the profile will still be reduced to a single measured value. The speed of measurement is sufficient for most terramechanics conditions which typically occur at very low vehicle speeds.
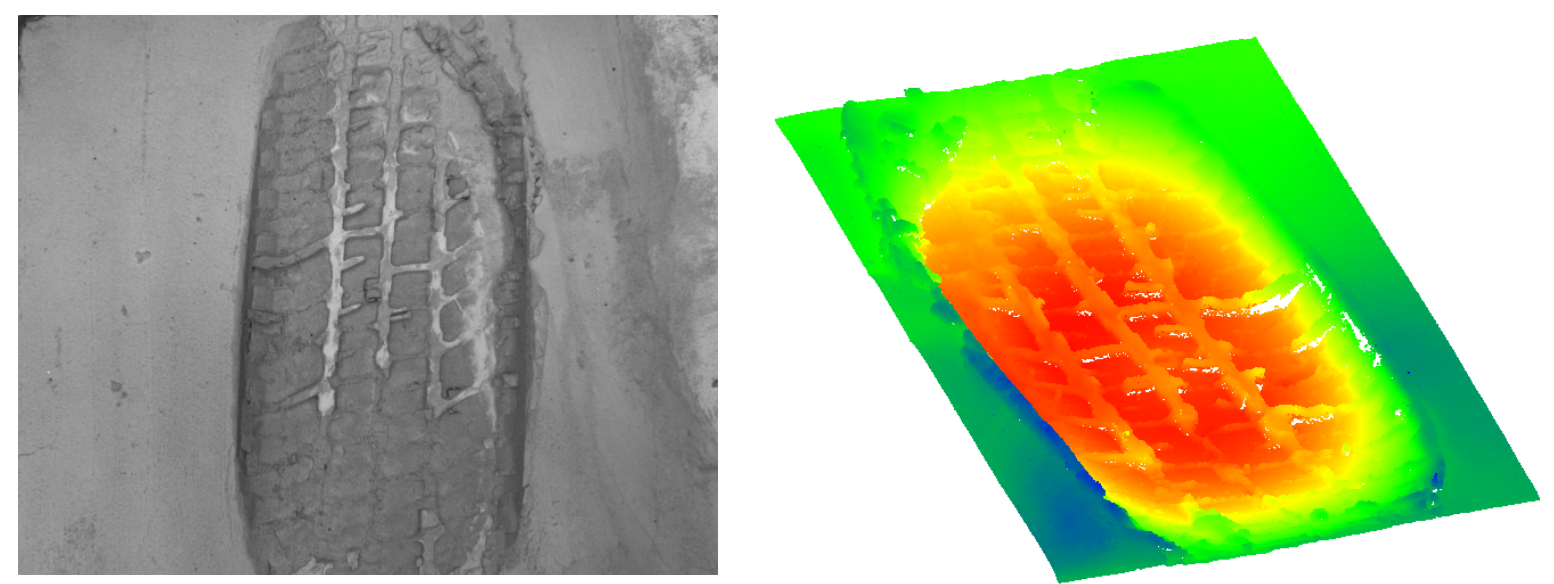

Fig. 2: Photo of reference rut formed in concrete(left) and point cloud of reference surface obtained from laser measurements(right) 


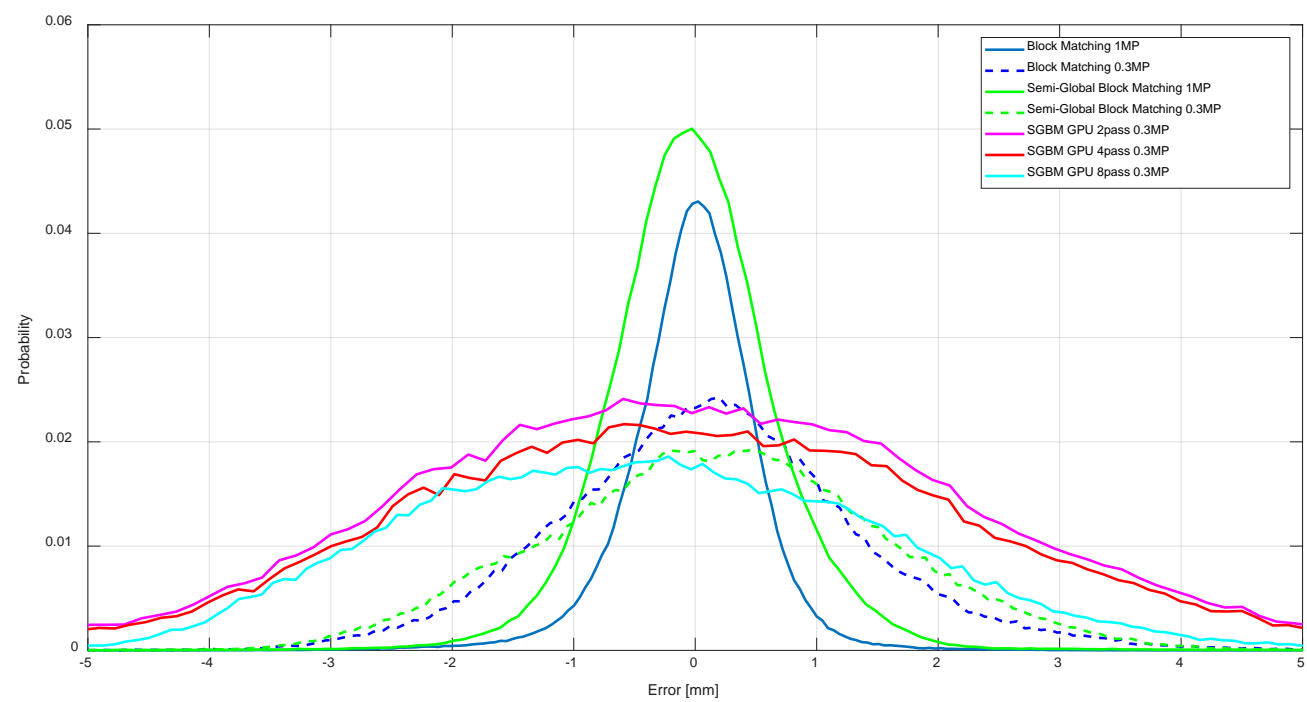

Fig. 3: Histogram error of the different algorithms

Table 2: Mean and standard deviation of algorithms

\begin{tabular}{|l|l|l|}
\hline Algorithm and Resolution & Mean [mm] & Standard Deviation [mm] \\
\hline BM 1MP & -0.022 & 0.544 \\
\hline BM 0.3MP & 0.117 & 1.254 \\
\hline SGBM 1MP & -0.019 & 0.730 \\
\hline SGBM 0.3MP & & 1.387 \\
\hline SGBM CUDA 2 pass 0.3MP & 0.183 & \\
\hline SGBM CUDA 4 pass 0.3MP & -0.347 & 4.890 \\
\hline SGBM CUDA 8 pass 0.3MP & -0.324 & 4.733 \\
\hline
\end{tabular}




\section{Experimental Testing on Various surfaces}

Tests were conducted to determine whether the system can be used to determine the depth of a scene on various types of surfaces which a vehicle may traffic. The tests were conducted by capturing synchronized images from the cameras mounted on different vehicles while simply driving over various types of deformable terrain. The images are post processed to determine the 3D surface profile of the terrain.

\subsection{Terrain Surface Profiles}

The terrain surface profiles of various types of terrains were determined to evaluate the system's ability to determine the profile on snow, ice, grass and mud. In the tests the same two PointGrey Flea3 cameras as in Section 3 were used but placed closer at $40 \mathrm{~mm}$ between cameras. The camera system was mounted on various vehicles in front and behind the lead tyres to measure the undeformed and deformed profile of different terrain. The cameras recorded at a resolution of $1280 \times 1024$ pixels, and the horizontal and lateral spatial resolution of the profiles obtained is dependent on the camera height but range between 0.3 to $1.0 \mathrm{~mm}$. The camera mounting locations on two vehicles are shown in Figure 4. The cameras were pointed directly towards the terrain approximately 400mm from the surface. The setups provided an overlap in the images of about $2 / 3$ of the image. A set of cameras were placed in front and behind the vehicles front tyres to measure undeformed as well as deformed tyres.
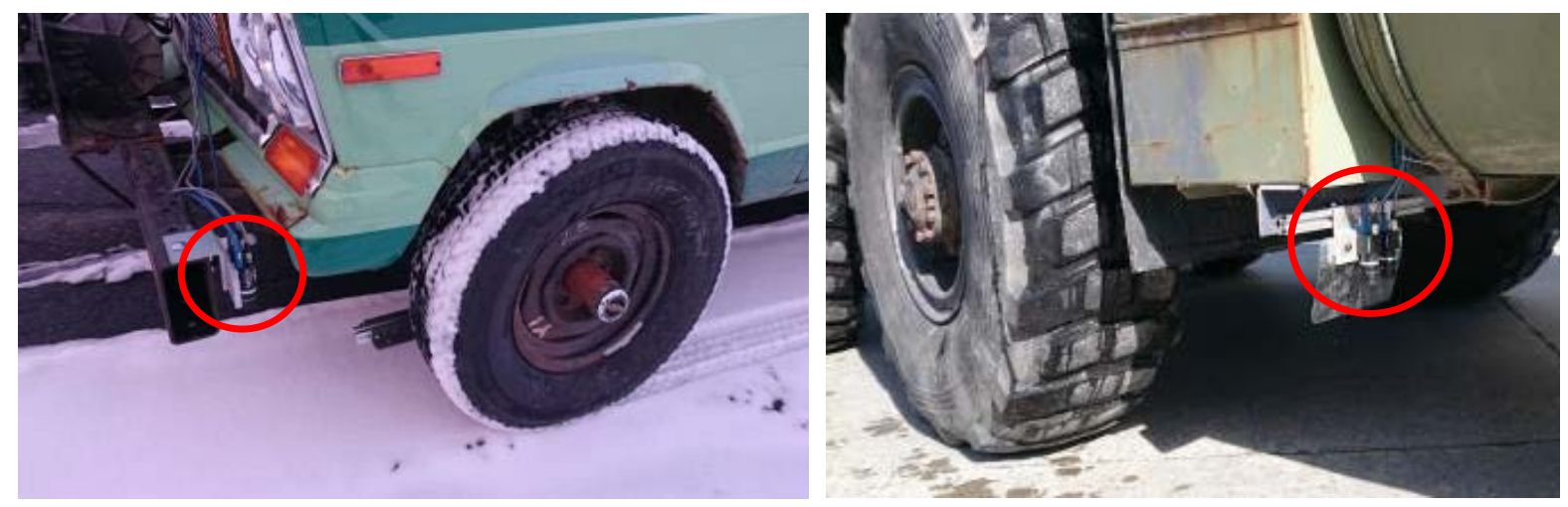

Fig. 4: Camera mounting position on vehicles. Cameras mounted in front of the tyre (left) and cameras mounted behind front tyres (right)

Figure 5 shows the camera image and surface profile of fresh undisturbed snow. The camera photo shows the lack of contrast in the image which can be considered one of the most challenging terrains to use stereo graphic profiling on. The surface profile however shows that the 3D profile of the surface can be determined and shows a 
fairly flat surface as expected. This shows that the techniques are capable of profiling even flat fresh snow. Figure 6 shows the camera image and surface profile of snow compacted by a single tyre pass. From the surface profile, the rut as well as tread indents are shown clearly. The rut depth was measured, using a ruler, to be $60 \mathrm{~mm}$ which corresponds to the rut depth in the surface profile. Figure 7 shows the profile of a mixture of cracked ice and water by two tyres passes of a large military vehicle using the stereo measurement system. The measured profile shows the larger indents in the surface caused by the larger lugs of the tyre from this vehicle. From this test it is evident that the surface of the water is profiled and not the actual terrain under the water. Thus, one limitation of this system is that water effectively interferes with the measurement of the soil surface.

Figure 8 shows the results from a grass-covered terrain after two tyre passes of a large military vehicle. The images again show the larger lug pattern. It is also evident that the grass is profiled as well showing some vertical elements sticking out from the profile. Therefore, if the grass-covered terrain does not conform to the ground profile then the grass will obscure the surface profile. Figure 9 shows a single tyre pass of a SUV vehicle on mud. This test clearly shows the rut formed by the tyre.

These tests demonstrate the ability to measure the profile of various terrains from fresh snow, which shows very little contrast, to terrains that show much more contrast such as grass, mud and deformed snow. The spatial resolution is also very high ( $<1 \mathrm{~mm}$ ) over large surfaces of $400 \mathrm{~mm}$ by $400 \mathrm{~mm}$ from just one set of images. Both the spatial resolution and surface dimensions can be varied based on camera lenses, camera height and camera resolution.
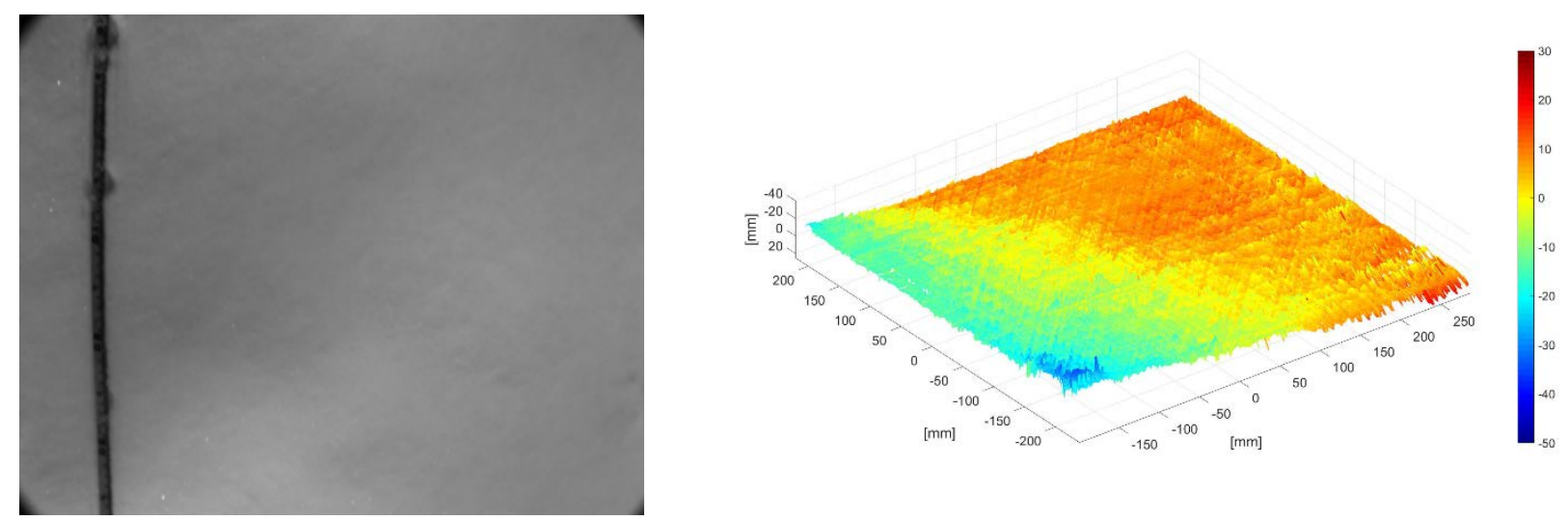

Fig. 5: Undisturbed fresh snow camera image (left) and corresponding surface profile (right) 

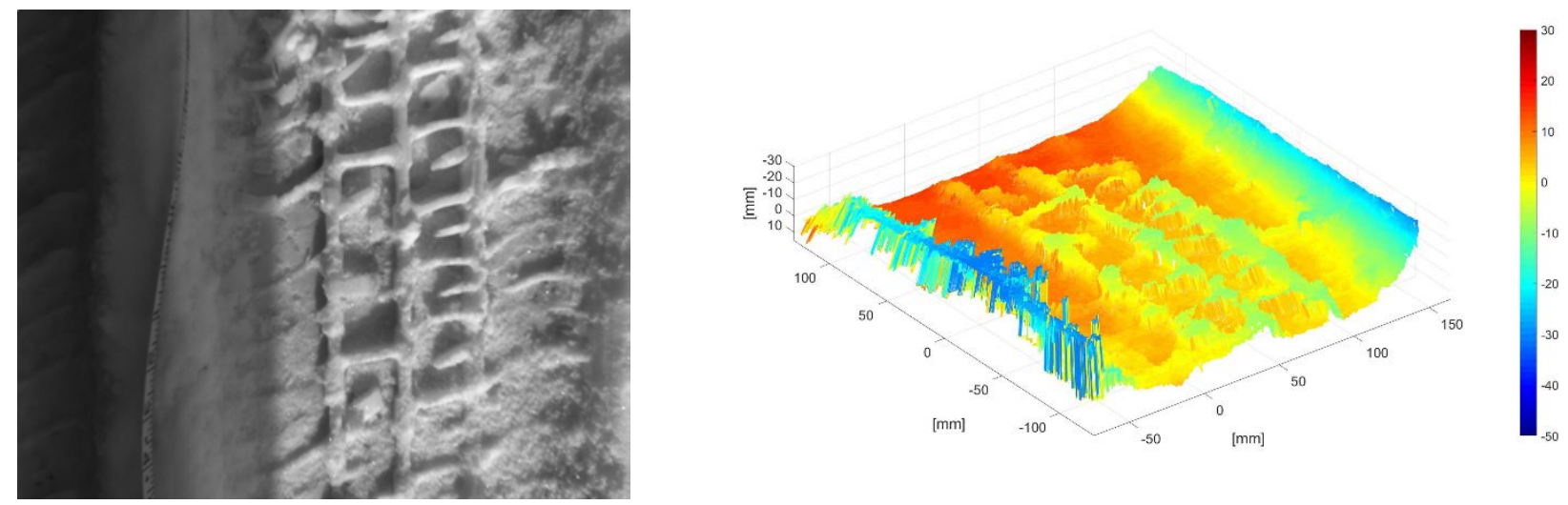

Fig. 6: Camera image of snow after single pass of a tyre (left) and corresponding surface profile (right)
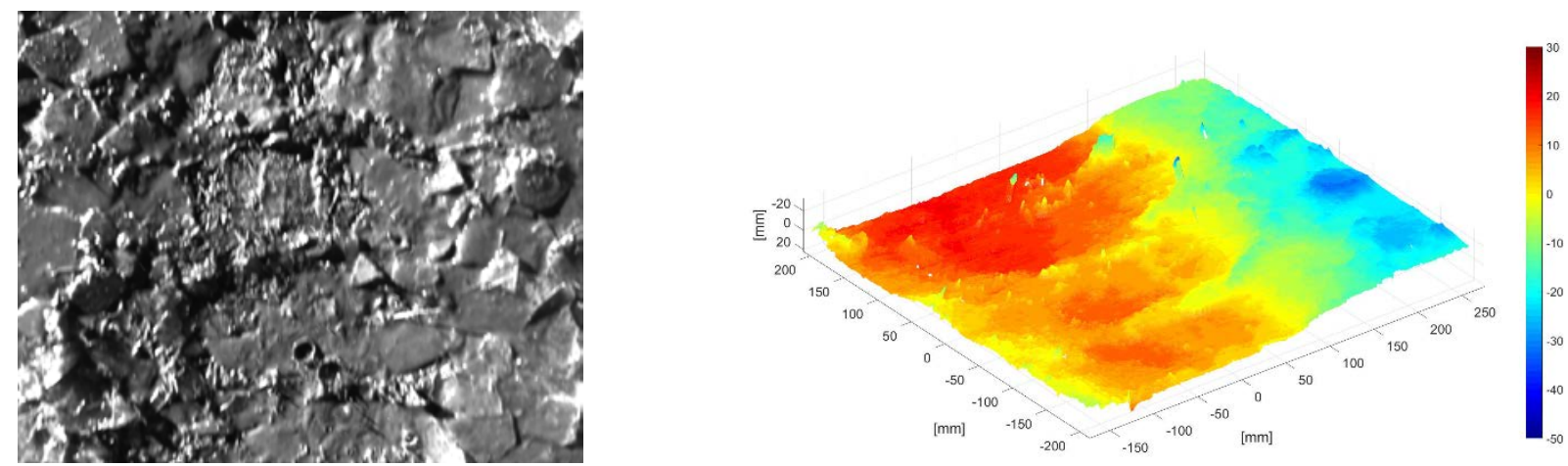

Fig. 7: Camera image of cracked ice/water (left) and corresponding surface profile (right)
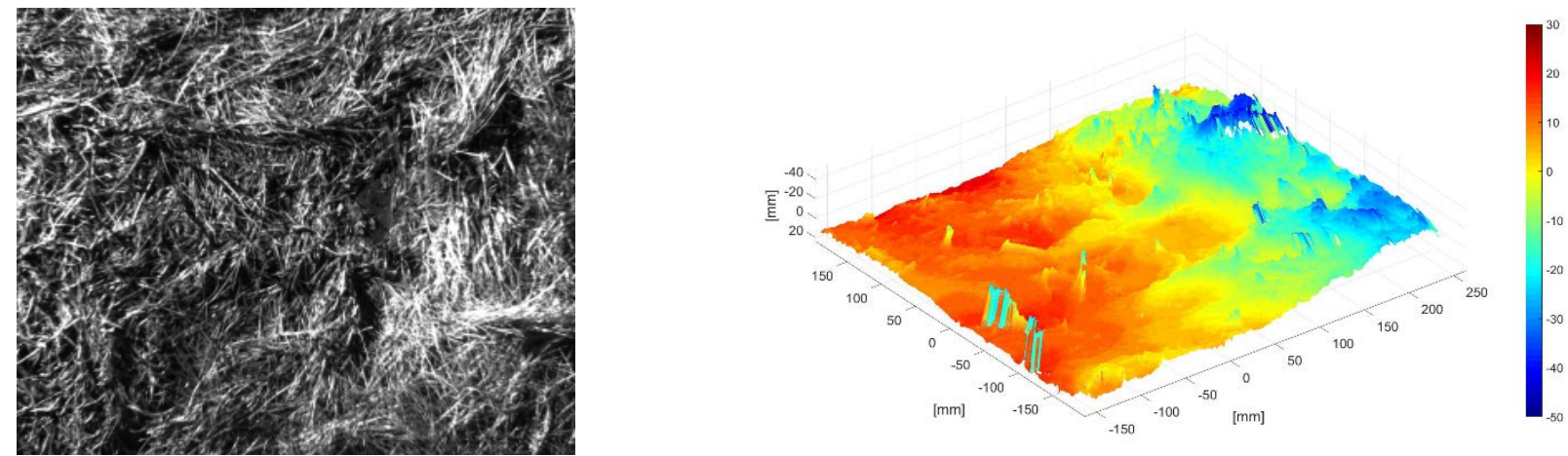

Fig. 8: Camera image of grass land after single pass of a tyre (left) and corresponding surface profile (right) 

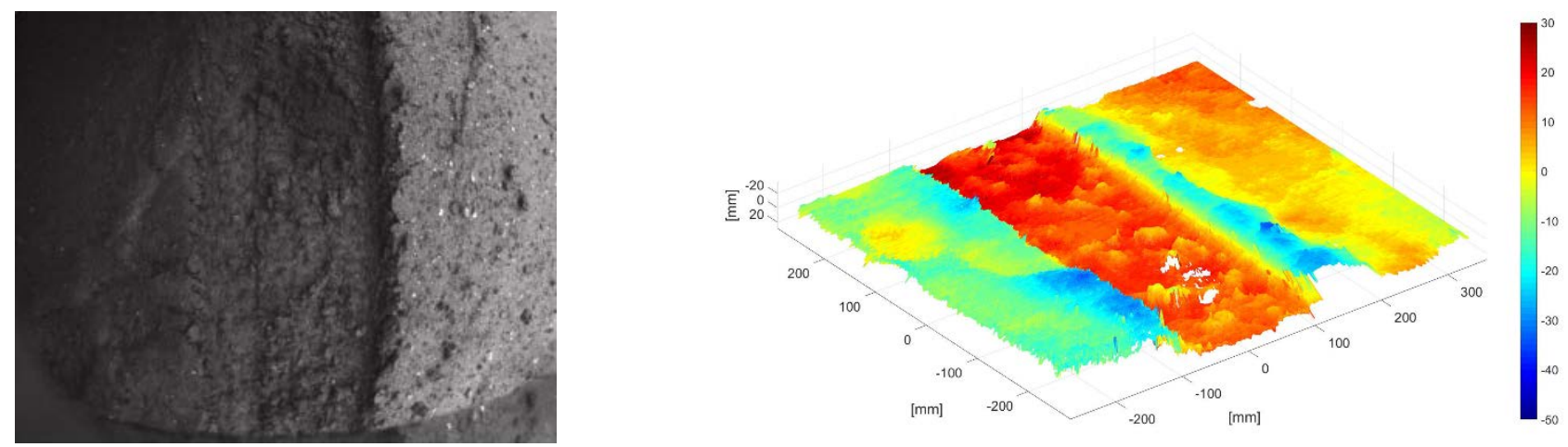

Fig. 9: Camera image of mud after single pass of a tyre (left) and corresponding surface profile (right)

\subsection{Automatic Rut Depth Measurement}

As the vehicle tyre rolls through deformable soil it displaces soil to the side of the tyre forming the rut which is a large indentation in the soil, as well as forming heaps or piles on the side. A cross section of a typical rut is shown in Figure 10 indicating the piles on either side of the rut, as well as, the rut depth measurement which is of interest.

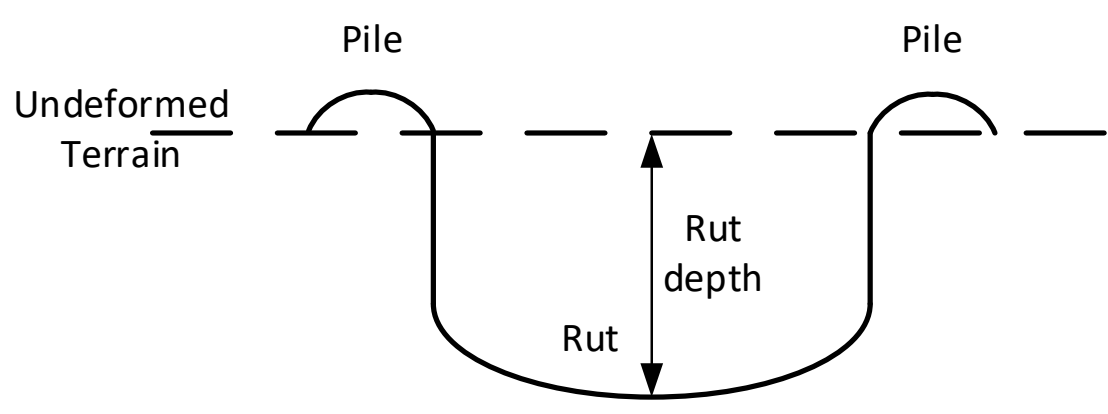

Fig. 10: Typical rut depth, showing rut and piles

The stereovision system will create a 3D representation of the rut in Figure 10. A 3D representation, from the BM CPU algorithm, is shown in Figure 11 and shows the same characteristics with piles on either side of the rut. In Figure 11 the vertical plot axis and colourbar denote the vertical distance from the camera principal point. Therefore, the distance to the rut is further away from the camera than the undeformed surface. In order to determine the rut depth, the distance to the deepest point of the rut from the reference undeformed surface needs to be determined. This requires that the location of the rut and the reference undeformed surface need to be determined. The location of the rut and undefromed surface can easily be determined once the location of the piles are known. Since the vehicle can negotiate turns or slide, and camera mounting locations may change, the pile 
locations will not be constant in the image. Therefore, an algorithm to automatically detect where the piles, undeformed terrain and rut are is required. The developed algorithm detects where in the 3D surface profile the piles are, and then can determine where the undeformed surface and the rut are in relation to the piles.

The first step of the algorithm reduces the surface profile to a 2D lateral cross-section of the surface profile. The 2D reduction is performed by averaging the height values by applying a box filter which slides laterally across the rut. The height at the centre of the box is taken as the average height across the box. This filter smooths the surface and removes noise.

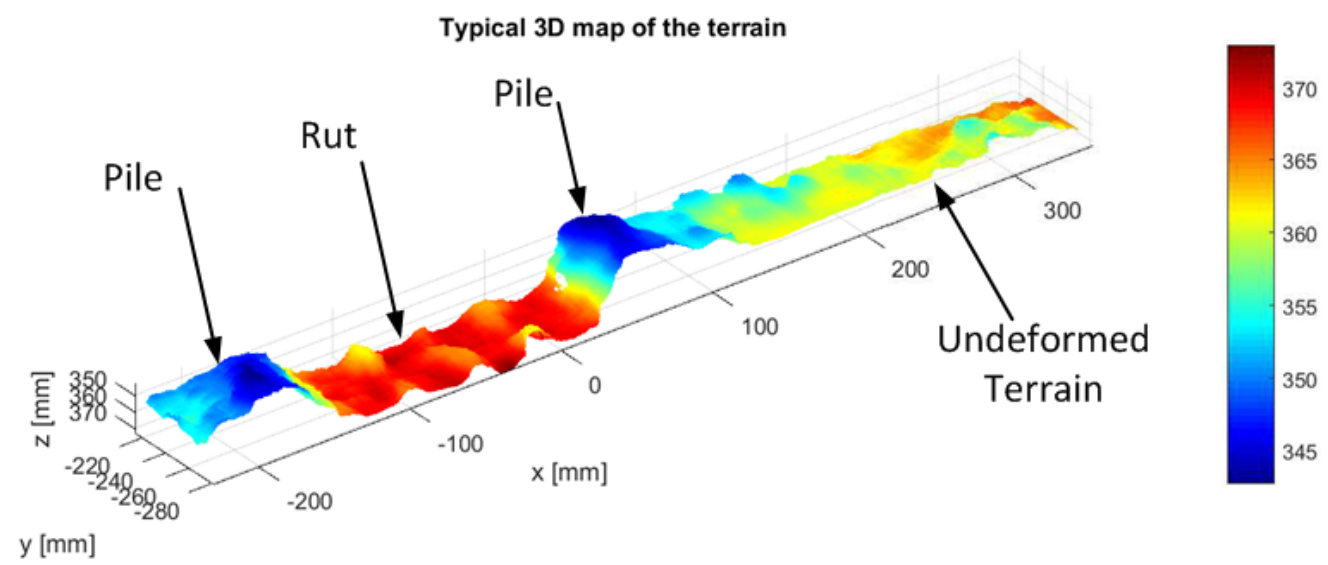

Fig. 11: Three dimensional cross-section of rut profile

The algorithm uses a gradient detection method to identify where the piles are since the pile locations will have a turning point in the middle of the pile where the piles is the highest. The gradient algorithm applies a parabola fit over a region of 50 pixels. If the parabola contains a turning point within the region the fit is applied to and the shape of the parabola indicates a minimum (since the piles will be convex as viewed from the camera perspective) then the point is saved in a vector. The region of which the parabolic fit is performed is moved across the $2 \mathrm{D}$ cross section of the rut until the process has been applied across the whole $2 \mathrm{D}$ profile. The turning points which represent the piles can be identified from the the saved vector of turning points. The piles are identified as the first two turning points which are within a specified distance from one another (as denoted by the tyre with). The points are also required to be closer to the camera than the average height of the profile. The piles also denote the start and end of the rut.

Once the start and end of the rut has been determined, points on either side of these locations, away from the rut, are used to determine the ground plane. Points with low gradients are used to fit a first order polynomial to. A first order polynomial, $y=m x+c$, is used to compensate for the surface which may not be perfectly level due 
to camera misalignment, vehicle roll or the surface which is not level. The rut is defined as the perpendicular distance from the reference line to the furthest point in the rut $\left(X_{p}, Y_{p}\right)$ :

$$
\text { RutDepth }=\frac{\left|X_{p} m-Y_{p}+c\right|}{\sqrt{1+m^{2}}}
$$

Figure 12 shows the output of the detection algorithm on a rut profile, note the vertical axis denotes the distance from the camera. The figure shows the ground reference plane which accurately detects the average height of the ground plane. The left pile is also well detected, the right pile is slightly inaccurate. This is due to the lack of points to the right hands side of the rut. The deepest point in the rut is also accurately detected. The automatic detection algorithm therefore works very well on a single 3D profile of a rut obtained from a single left and right image from the calibrated stereovision system

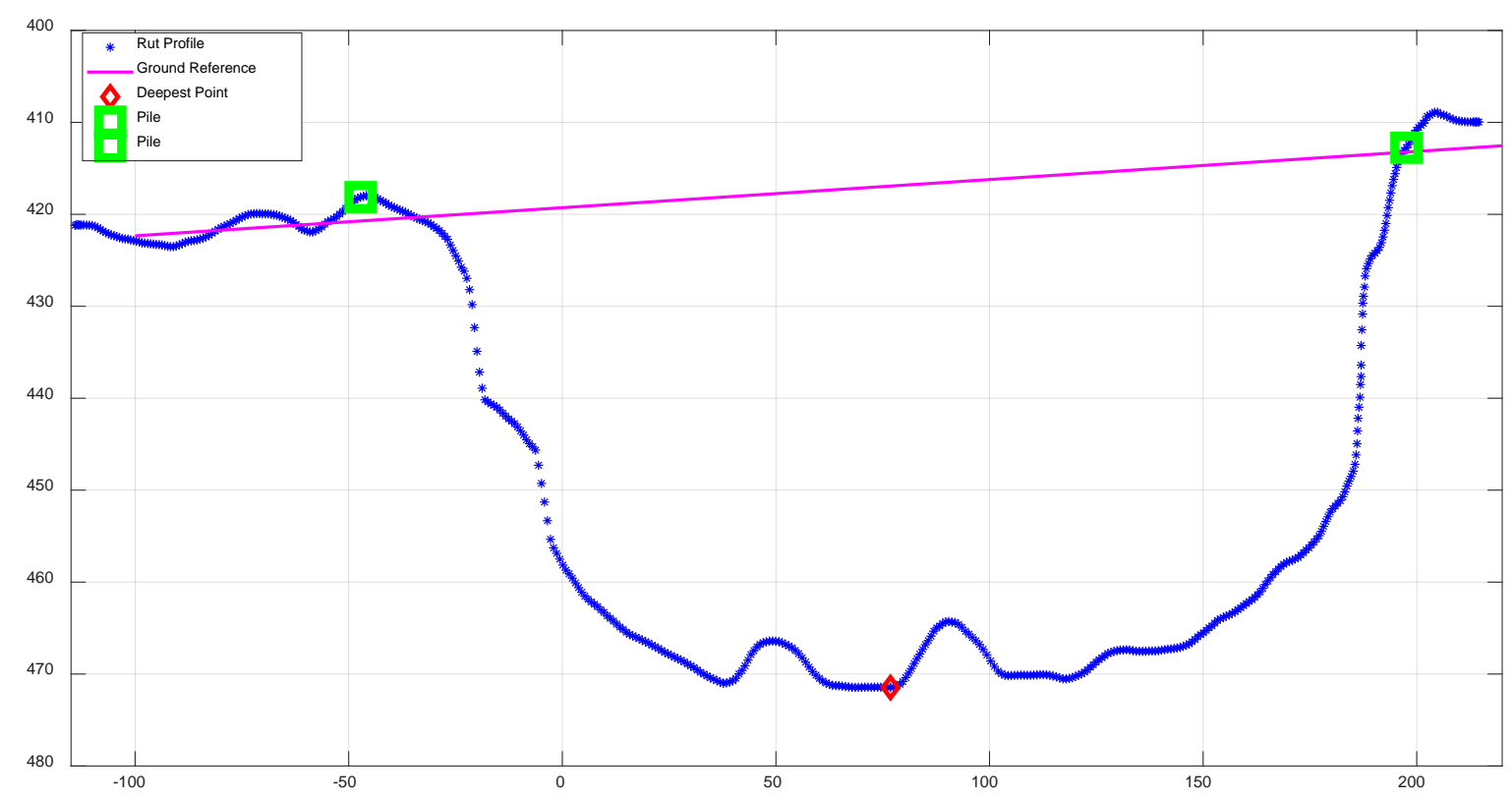

Fig. 12: Rut depth measurement algorithm showing rut, ground plane, furthest point and gradient of rut

The accuracy of the algorithm is tested by using different rut depths which were cast in concrete. Four different depths were created of roughly 30, 40, 50 and 60mm. The same camera setup as used in Section 3.2 is used to measure the depth in the centre of the rut. The rut depth was also manually measured using a Vernier, the results of the measurements are shown in Table 3 . The results show that the measurements are within $2 \mathrm{~mm}$ of the 
manual measurements. It should be noted that manually identifying the deepest point in the rut is not necessarily the most accurate method either. The algorithm is also very efficient and capable of running at $100 \mathrm{~Hz}$ on a 640x480 pixel image running on a mid-range CPU (i5-570, 2.7GHz). Therefore, the overall sampling frequency of the systems is mainly dominated by the time to perform the disparity mapping. The time to derermine the depth of the rut is not as computationally expensive

Table 3: Manual and automatic measurement of rut depth comparison

\begin{tabular}{|l|l|l|}
\hline Manufactured Depth [mm] & Measurement from Algorithm [mm] & Manual Measurement [mm] \\
\hline 30 & 26.76 & 27 \\
\hline 40 & 44.66 & 43 \\
\hline 50 & 48.87 & 47 \\
\hline 60 & & 59 \\
\hline
\end{tabular}

The above analysis was only conducted on single images of ruts. The system is however intended to continually operate on a sequence of images. In order to test the algorithm in continually detecting the rut depth a video recording of an SUV driving through mud is used. During the test only a few points were measured by means of a vernier. Therefore, in order to validate the rut detection algorithm, the rut depth of the automatic detection algorithm is compared to the depth where the location of the rut and the underformed terrain on the 3D profile was manually selected on the image. The same reference line was fitted to the manually selected regions which contained undeformed terrain. The furthest point from the reference line in the manually selected rut region is also identified using the same process to obtain the rut depth. The result of the automatically detected algorithm are compared to the manually selected region are shown in Figure 13.The figure also shows the error between the two measurements. The results show that overall the two depth measurements are very close to one another with a few points of the automatic detection algorithm deviating from the manual measurements. The average error over the whole test is $1.6 \mathrm{~mm}$. This indicates that the system can automatically detect the rut and accurately 
measure the rut depth in real time. The real time performance can be altered by changing the disparity mapping algorithm, this will however also affect the accuracy of the system.
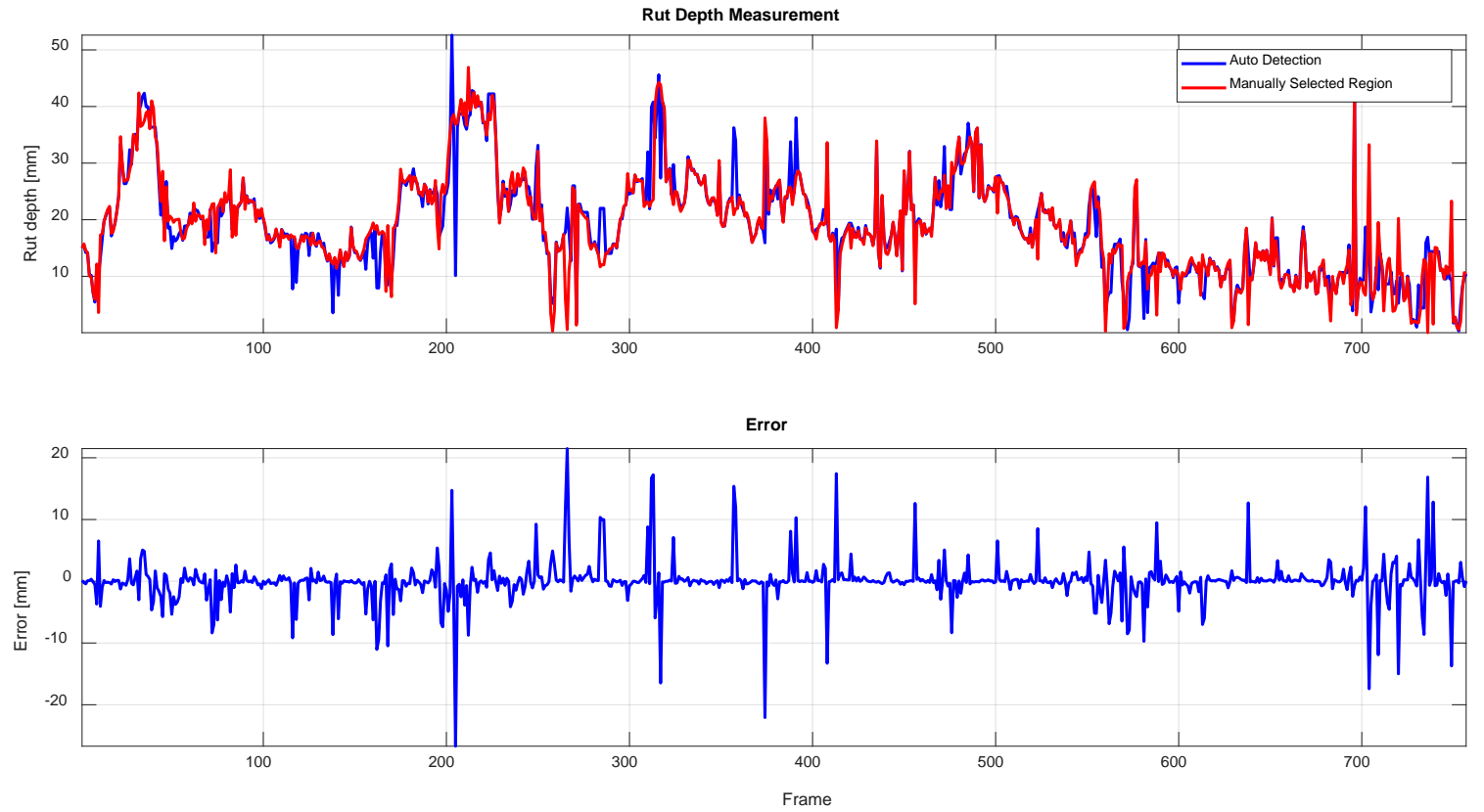

Fig. 13: Continual rut depth detection validation of algorithm.

The main disadvantage of the system is that it is optically based. This means that obstruction of the lenses can result in the system being incapable of making any measurements. This can be prevented by mounting the system strategically to prevent soil being kicked up into the system. The system also requires suitable lighting to be able to capture images which are not blurred. This can be a problem at low light conditions such as from late afternoon. However, this can easily be corrected for by using artificial lighting by means of a lighting source. 


\section{Conclusion}

This paper evaluated the feasibility of using cameras in a calibrated stereo rig to determine the rut depth of surface profiles of various terrains in real time using off the shelf cameras and hardware. Various disparity mapping algorithms are evaluated to determine the accuracy and real time capability of the algorithms. The various algorithms can provide good accuracy (within $1.5 \mathrm{~mm})$ at lower sampling speeds $(15 \mathrm{~Hz})$ and reduced accuracy (5mm) at very high speed $(58 \mathrm{~Hz})$.

The camera system was mounted on various vehicles in front and behind the lead tyres to measure the undeformed and deformed profile of different terrain. The system has shown measurement capability on fresh snow, deformed snow, ice, grassland as well as mud. An efficient algorithm was developed which can determine the rut depth automatically from a sequence of images.

The main disadvantage of the system is that it is optically based requiring a good lighting and unobstructed view of the terrain. This can be solved by using artificial lighting and mounting the cameras in strategic places.

The real time measurements of rut depth can be used for mobility under off-road conditions that can change rapidly due to environmental conditions e.g. rain or snow, complicating the driver's task. The techniques described can assist in gathering terrain and vehicle mobility data during driving that can be used directly to assist the driver in making safety related decisions.

\section{References}

Anderson, A.B., Palazzo, A.J., Ayers, P.D., Fehmi, J.S., Shoop, S. and Sullivan, P., 2005. Assessing the impacts of military vehicle traffic on natural areas. Introduction to the special issue and review of the relevant military vehicle impact literature. Journal of Terramechanics, 42(3-4), pp.143-158.

Besl, P.J. and McKay, N.D., 1992, April. Method for registration of 3-D shapes. In Sensor Fusion IV: Control Paradigms and Data Structures (Vol. 1611, pp. 586-607). International Society for Optics and Photonics.

Botha, T.R. and Els, P.S., 2015a. Digital image correlation techniques for measuring tyre-road interface parameters: Part 1-Side-slip angle measurement on rough terrain. Journal of Terramechanics, 61, pp.87-100.

Botha, T.R. and Els, P.S., 2015b. Digital image correlation techniques for measuring tyre-road interface parameters: Part 2-Longitudinal tyre slip ratio measurement. Journal of Terramechanics, 61, pp.101-112. 
Botha, T.R. and Els, P.S., 2015c. Rough terrain profiling using digital image correlation. Journal of Terramechanics, 59, pp.1-17.

Halvorson, J.J., McCool, D.K., King, L.G. and Gatto, L.W., 2001. Soil compaction and over-winter changes to tracked-vehicle ruts, Yakima Training Center, Washington. Journal of Terramechanics, 38(3), pp.133-151.

Hemmat, A., Yaghoubi-Taskoh, M., Masoumi, A. and Mosaddeghi, M.R., 2014. Relationships between rut depth and soil mechanical properties in a calcareous soil with unstable structure. biosystems engineering, 118, pp.147155.

Hernandez-Juarez, D., Chacón, A., Espinosa, A., Vázquez, D., Moure, J.C. and López, A.M., 2016. Embedded real-time stereo estimation via semi-global matching on the GPU. Procedia Computer Science, 80, pp.143-153.

Herrick, J.E. and Jones, T.L., 2002. A dynamic cone penetrometer for measuring soil penetration resistance. Soil Science Society of America Journal, 66(4), pp.1320-1324.

Hirschmüller, H., 2005, Accurate and efficient stereo processing by semi-global matching and mutual information. In Computer Vision and Pattern Recognition, 2005. CVPR 2005. IEEE Computer Society Conference on IEEE (Vol. 2), pp. 807-814)..

Iagnemma, K., Kang, S., Brooks, C. and Dubowsky, S., 2003. Multi-sensor terrain estimation for planetary rovers. In Proceedings of the 8th international symposium on artificial intelligence, robotics, and automation in space. IEEE Press, New York.

Kenarsari, A.E., Vitton, S.J. and Beard, J.E., 2017. Creating 3D models of tractor tire footprints using close-range digital photogrammetry. Journal of Terramechanics, 74, pp.1-11.

Konolige, K., 1998. Small vision systems: Hardware and implementation. In Robotics Research (pp. 203-212). Springer, London.

Kurjenluoma J, Alakukkua L, Ahokas J. 2009 Rolling resistance and rut formation by implement tyres on tilled clay soil. Journal of Terramechanics 46(6), pp. 267-275 
Pierzchała, M., Talbot, B. and Astrup, R., 2014. Estimating soil displacement from timber extraction trails in steep terrain: application of an unmanned aircraft for 3D modelling. Forests, 5(6), pp.1212-1223.

Pierzchała, M., Talbot, B. and Astrup, R., 2016. Measuring wheel ruts with close-range photogrammetry. Forestry: An International Journal of Forest Research, 89(4), pp.383-391.

Salmivaara, A., Miettinen, M., Finér, L., Launiainen, S., Korpunen, H., Tuominen, S., Heikkonen, J., Nevalainen, P., Sirén, M., Ala-Ilomäki, J. and Uusitalo, J., 2018. Wheel rut measurements by forest machine-mounted LiDAR sensors-accuracy and potential for operational applications?. International Journal of Forest Engineering, pp.112.

Shibly H, Iagnemma K, and Dubowsky S. 2005. An equivalent soil mechanics formulation for rigid wheels in deformable terrain, with application to planetary exploration rovers. Journal of Terramechanics; 42(1), pp. 1-13 Shoop, S., Knuth, M. and Wieder, W., 2013. Measuring vehicle impacts on snow roads. Journal of Terramechanics, 50(1), pp.63-71.

Shoop, S.A., Coutermarch, B., Ayers, P., Anderson, A. and Affleck, R. 2008. Tire Force and Terrain Disturbance Measurements During Turning Maneuvers. American Society of Agricultural and Biological Engineers ISSN 0001-2351. 51(6), pp. 1869-1878

Siebert, S. and Teizer, J., 2014. Mobile 3D mapping for surveying earthwork projects using an Unmanned Aerial Vehicle (UAV) system. Automation in Construction, 41, pp.1-14.

Soane, B.D., Blackwell, P.S., Dickson, J.W. and Painter, D.J., 1980. Compaction by agricultural vehicles: A review I. Soil and wheel characteristics. Soil and Tillage Research, 1, pp.207-237.

Soane, B.D. and Van Ouwerkerk, C., 1995. Implications of soil compaction in crop production for the quality of the environment. Soil and Tillage Research, 35(1-2), pp.5-22. 